Е.И. Новикова, В.К. Боженко, Е.А. Кудинова, В.А. Солодкий

\section{Исследование влияния генетического варианта с.470T>C в гене CHEK2 на повышение риска развития рака молочной железы у населения Российской Федерации}

\author{
ФГБУ «Российский научный центр рентгенорадиологии» Минздрава России; Россия, 117997 Москва, ул. Профсоюзная, 86
}

Конта кты : Екатерина Ивановна Новикова e.novikova.rncrr@mail.ru

\begin{abstract}
Введение. В настоящее время имеются противоречивые данные относительно влияния герминальной мутации с.470T>C в гене CHEK2 на повышение риска возникновения рака молочной железы (РМЖ), поэтому необходимы исследования на больших выборках больных, в том числе в российской популяции, в целях анализа вклада данной мутации в риск развития онкологического заболевания.

Цель исследования - определение частоты встречаемости генетического варианта с.470T>C в гене CHEK2 в российской популяции у больных РМЖ и пациенток с доброкачественными заболеваниями молочной железы (ДЗМЖ) для оценки возможного влияния данного повреждения дезоксирибонуклеиновой кислоты на вероятность возникновения онкологического заболевания.

Материалы и методы. В исследование были включены 2787 больных РМЖ и 1004 пациентки с ДЗМЖ, проходившие обследование и лечение в ФГБУ «Российский научный центр рентгенорадиологии» Минздрава России с 2010 по 2018 г. Молекулярно-генетическое исследование для определения характерного для российской популяции наследственного генетического варианта с.470Т >C в гене СНЕК2 было проведено методом полимеразной цепной реакции в режиме реального времени с использованием диагностической панели, позволяющей определять три герминальные мутации: c.1100delC, c.444+1G>A и c.470T>C в гене CHEK2.

Результаты. У больных с диагнозом РМЖ частота мутации с.470Т >C в гене СНЕК2 составила 3,8 \%, у пациенток с ДЗМЖ данная мутация выявлена в 4,7 \% случаев. Частота генетического варианта с.470Т>C в группах повышенного риска составила 5,1 \% для больных РМЖ с клиническими признаками наследственного заболевания и 4,9\% для пациенток с ДЗМЖ, имеющих онкологически отягощенный семейный анамнез. Статистически значимых различий между частотой мутации с.470Т>C в общих группах больных РМЖ и пациенток с ДЗМЖ и соответствующей частотой в группах повышенного риска, а также в группах больных РМЖ и пациенток с ДЗМЖ не установлено $(p>0,05)$.

Заключение. Результаты проведенного исследования свидетельствуют о вероятном отсутствии связи между наличием мутации с.470T>C в гене CHEK2 и повышением риска развития РМЖ.
\end{abstract}

Ключевые слова: наследственный рак молочной железы, мутации в гене CHEK2, генетический вариант с.470T>C

Для цитирования: Новикова Е.И., Боженко В.К., Кудинова Е.А., Солодкий В.А. Исследование влияния генетического варианта с.470T>C в гене CHEK2 на повышение риска развития рака молочной железы у населения Российской Федерации. Успехи молекулярной онкологии 2021;8(1):26-31. DOI: 10.17650/2313-805X-2021-8-1-26-31.

\title{
Studying of the effect of the genetic variant c.470T>C in the CHEK2 gene on increasing the risk of breast cancer in the population of the Russian Federation
}

\author{
E.I. Novikova, V.K. Bozhenko, E.A. Kudinova, V.A. Solodkiy \\ Russian Scientific Center of Roentgenoradiology, Ministry of Health of Russia; 86 Profsoyuznaya St., Moscow 117997, Russia
}

Con t a c t s : $\quad$ Ekaterina Ivanovna Novikova e.novikova.rncrr@mail.ru

Introduction. Currently, there are conflicting data regarding the effect of the c.470T> C germline mutation in the CHEK2 gene on increasing the risk of breast cancer (BC), so it is necessary to conduct research on large samples of patients, including in the Russian population, in order to analyze the contribution of this mutation to the risk of cancer developing. 
The aim of the study was to determine the frequency of occurrence of the genetic variant c.470T>C in the CHEK2 gene in the Russian population in patients with $B C$ and patients with benign breast diseases (BBD) to assess the possible effect of this deoxyribonucleic acid damage on the likelihood of cancer occurrence.

Materials and methods. The study included 2,787 patients with BC and 1,004 patients with BBD who underwent examination and treatment at the Russian Scientific Center of Roentgenoradiology of the Ministry of the Russian Federation from 2010 to 2018 . Molecular genetic study was carried out by real-time polymerase chain reaction to determine the characteristic of the Russian population hereditary genetic variant c.470T $>\mathrm{C}$ in the CHEK2 gene using a diagnostic panel that allows to determine three germline mutations: c.1100delC, c.444+1G >A and c.470T>C in the CHEK2 gene. Results. In patients with $B C$ the frequency of the mutation c.470T $>C$ in the $C H E K 2$ gene was $3.8 \%$, in patients with BBD this mutation was detected in $4.7 \%$ of cases. The frequency of the genetic variant c.470T $>C$ in high-risk groups was: $5.1 \%$ - for BC patients with clinical signs of hereditary disease and $4.9 \%$ - for patients with BBD with a family history of cancer. There were no statistically significant differences between the frequency of the mutation c.470T>C in the general groups of $\mathrm{BC}$ patients and patients with $\mathrm{BBD}$ and the corresponding frequency in the high-risk groups, as well as in the groups of BC patients and patients with $\operatorname{BBD}(p>0.05)$.

Conclusion. The results of this study indicate the probable absence of a relationship between the presence of the mutation C.470T >C in the CHEK2 gene and an increased risk of BC.

Key words: hereditary breast cancer, mutations in the CHEK2 gene, genetic variant c.470T>C

For citation: Novikova E.I., Bozhenko V.K., Kudinova E.A., Solodkiy V.A. Studying of the effect of the genetic variant C.470T>C in the CHEK2 gene on increasing the risk of breast cancer in the population of the Russian Federation. Uspekhi molekulyarnoy onkologii = Advances in Molecular Oncology 2021;8(1):26-31. (In Russ.) DOI: 10.17650/2313805X-2021-8-1-26-31.

\section{ВВЕДЕНИЕ}

Ген СНEK2 находится на втором месте по частоте встречаемости в нем наследственных мутаций при раке молочной железы (РМЖ) после генов BRCA1 и $B R C A 2$ в российской популяции. $C H E K 2$ кодирует треонин киназу (threonine kinase) - белок, который активируется при повреждении дезоксирибонуклеиновой кислоты (ДНК) и участвует в процессах репарации, клеточной пролиферации, а также контролирует процессы апоптоза [1]. Поэтому риск развития онкологического заболевания у носителей повреждений в данном гене повышен по сравнению с общепопуляционным. Для российской популяции наиболее характерны герминальные мутации с.1100delC, c.444+1G>A и с. $470 \mathrm{~T}>\mathrm{C}$ в гене $C H E K 2$, которые были внесены в стандартные диагностические панели, использующиеся в ежедневной практике молекулярно-генетических лабораторий [2]. Но эти генетические повреждения имеют разную пенетрантность, т. е. вероятность развития заболевания у носителей мутаций различается. Если связь между наличием мутаций с.1100delC и с. $444+1 \mathrm{G}>\mathrm{A}$ в гене $C H E K 2$ и повышением риска развития РМЖ у их носителей в 3-5 раз доказана [3-5], то относительно связи генетического варианта с.470Т $>$ C с повышением риска развития РМЖ имеются противоречивые данные. Часть проведенных зарубежных исследований описывает достоверное, хоть и небольшое повышение риска (отношение шансов (ОШ) $=1,4-1,58$ ) у носителей данной мутации [5-8]. В других научных работах, в том числе российских, связь с повышенным риском развития (ПРР) РМЖ опровергается [2, 9-12]. В настоящее время даже для носителей среднепенетрантных мутаций с. $1100 \mathrm{delC}$ и с.444+1G $>$ А в гене $C H E K 2$, а уж тем более для пациентов, имеющих низкопенетрантный генетический вариант с.470T $>\mathrm{C}$, от- сутствуют конкретные рекомендации по профилактике и лечению, кроме проведения диагностических мероприятий (маммография в сочетании с магнитно-резонансной томографией по достижению 30-40 лет) [13]. Однако ведущие российские онкологи учитывают наличие данной мутации (с.470T>C) при выборе тактики лечения и проведении профилактических операций [14]. В связи с этим исследования на больших выборках больных РМЖ в российской популяции для изучения вклада данного варианта в риск развития онкологического заболевания являются актуальными и клинически важными.

\section{МАТЕРИАЛЫ И МЕТОДЫ}

В молекулярно-генетическое исследование были включены 2787 пациенток с диагнозом «РМЖ», которым он был впервые поставлен в возрасте от 20 до 90 лет (средний возраст манифестации заболевания 58 лет), и 1004 пациентки с доброкачественными заболеваниями молочной железы (ДЗМЖ), у которых болезнь развилась в возрасте от 20 до 78 лет (средний возраст ее манифестации - 45 лет).

Пациентки проходили обследование и лечение в ФГБУ «Российский научный центр рентгенорадиологии» Минздрава России с 2010 по 2018 г. (табл. 1).

Среди пациенток были выделены группы ПРР заболевания, согласно рекомендациям Европейского общества медицинской онкологии (European Society for Medical Oncology, ESMO) [15] и Национальной онкологической сети США (National Comprehensive Cancer Network, NCCN) [13]. В данные группы вошли 486 пациенток с ДЗМЖ, имеющих онкологически отягощенный семейный анамнез, и 1072 больные РМЖ, имеющих хотя бы один из следующих клинических признаков наследственного заболевания (КПНЗ): 
Таблица 1. Клиническая характеристика пациентов, включенных в молекулярно-генетическое исследование

Table 1. Clinical characteristics of patients included in the molecular genetic examination

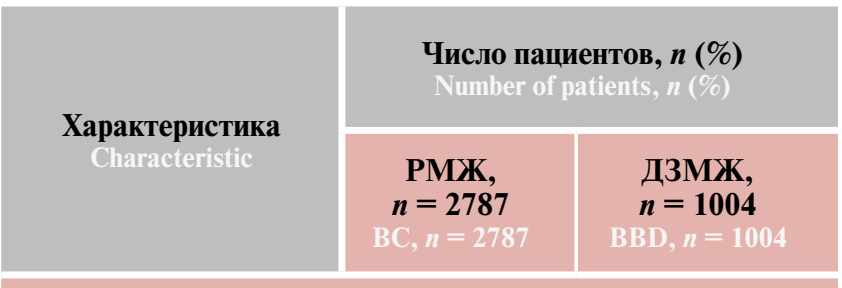

\section{1. Возраст}

1. Age

Средний возраст манифестации заболевания Average age of disease $58(20-90)$ лет $42(20-78)$ года manifestation

\begin{tabular}{|l|l|l|}
\hline $\begin{array}{l}\text { До } 50 \text { лет } \\
\text { Under } 50 \text { у.о. }\end{array}$ & $975(35)$ & $823(82)$ \\
\hline $\begin{array}{l}51 \text { год и старше } \\
51 \text { у.о. and older }\end{array}$ & $1812(65)$ & $181(18)$ \\
\hline
\end{tabular}

51 y.o. and older

2. Семейный онкологический анамнез 2. Pamily cancer history

\begin{tabular}{|l|r|r|}
\hline $\begin{array}{l}\text { Отягощен } \\
\text { Burden }\end{array}$ & $362(13)$ & $486(48)$ \\
\hline $\begin{array}{l}\text { Не отягощен } \\
\text { Not burden }\end{array}$ & $2425(87)$ & $518(52)$ \\
\hline & $\begin{array}{l}\text { 3. Диагно3 } \\
\text { 3. Diagnosis }\end{array}$ \\
\hline
\end{tabular}

\section{ПМЗН (РМЖ/РМЖ} или РМЖ/РЯ)

PMMN (BC/BC or BC/

$\mathrm{OC})$

РМЖ

$\mathrm{BC}$

157 (6)

$2630(94)$

Фиброаденома

молочной железы

Breast fibroadenoma

Солитарная киста молочной железы

Breast solitary cyst

Диффузная кистозная мастопатия

Diffuse cystic mastopathy

Фиброаденоз

молочной железы

Breast fibroadenosis

Фибросклероз молочной железы

Breast fibrosclerosis

Другие доброкачественные дисплазии молочной железы Other benign breast dysplasias
Окончание табл. 2

\begin{tabular}{|c|c|c|}
\hline \multicolumn{3}{|c|}{$\begin{array}{l}\text { 4. Молекулярный подтип опухоли } \\
\text { 4. Molecular subtype of tumor }\end{array}$} \\
\hline $\mathrm{ER}^{+}$и/или $\mathrm{PR}^{+}, \mathrm{Her} 2^{-}$ & $1784(64)$ & - \\
\hline $\mathrm{ER}^{+}$и/или $\mathrm{PR}^{+}, \mathrm{Her} 2^{+}$ & $613(22)$ & - \\
\hline $\mathrm{ER}^{-}, \mathrm{PR}^{-}, \mathrm{Her} 2^{+}$ & $139(5)$ & - \\
\hline $\mathrm{ER}^{-}, \mathrm{PR}^{-}, \mathrm{Her} 2^{-}$ & $251(9)$ & - \\
\hline
\end{tabular}

\section{5. Гистологический тип опухоли} . Histological type of tumor

Инвазивный протоко-

вый РМЖ

Infiltrating ductal

$1951(70)$

carcinoma

Инвазивный долько-

вый РМЖ

$446(16)$

Invasive lobular carcinoma

Другие

Other

$390(14)$

\section{6. Признак повышенного риска развития РМЖ}

6. Sign of an increased risk of breast cancer developing

Наличие

Presence

$1072(38)$

$486(48)$

Отсутствие

Absence

$1715(62)$

$518(52)$

Примечание. ДЗМЖ - доброкачественные заболевания молочной железы; ПМЗН - первично-множественные злокачественные новообразования; РМЖ - рак молочной железы; РЯ - рак яичников.

Note. $B B D$ - benign breast diseases; PMMN - multiple primary malignant neoplasms; $B C$ - breast cancer; $O C$ - ovarian cancer.

молодой возраст манифестации заболевания (до 50 лет), онкологически отягощенный семейный анамнез (наличие родственников первой и/или второй степени родства с диагнозом РМЖ и/или рака яичников (РЯ)), наличие первично-множественных опухолей (РМЖ/РМЖ или РМЖ/РЯ) (см. табл. 1).

ДНК была выделена из 100 мкл периферической крови с помощью наборов «М-Сорб» (компания «Синтол», Россия) в соответствии с протоколом производителя.

Постановку полимеразной цепной реакции в режиме реального времени проводили с использованием наборов реагентов «ОнкоГенетика СНЕК2» (ООО «НПФ ДНК-Технология», Россия) на детектирующем амплификаторе «ДТ-96» (ООО «НПО ДНКТехнология», Россия) согласно инструкции, предоставленной фирмой-производителем.

Полученные данные обрабатывались с помощью программного обеспечения StatSoft Statistica 10.0. Для сравнения частот мутации в различных группах пациенток применялся анализ таблиц сопряженности 
Таблица 2. Частота мутации с.470Т >С в гене СНЕК2 у больных раком молочной железы (РМЖ) и пациенток с доброкачественными заболеваниями молочной железы (ДЗМЖ)

Table 2. Frequency of the c.470T $>$ C mutation in the CHEK2 gene in patients with breast cancer (BC) and patients with benign breast diseases (BBD)

\begin{tabular}{|c|c|c|c|c|}
\hline \multirow{2}{*}{$\begin{array}{l}\text { Диагноз } \\
\text { Diagnosis }\end{array}$} & \multicolumn{2}{|c|}{$\begin{array}{l}\text { Число пациентов с мутацией, } \boldsymbol{n} \\
\text { Number of mutation carriers, } n\end{array}$} & \multicolumn{2}{|c|}{$\begin{array}{l}\text { Частота мутации, \% } \\
\text { Mlutation frequency, \% }\end{array}$} \\
\hline & $\begin{array}{l}\text { Общая группа } \\
\text { General group }\end{array}$ & $\begin{array}{l}\text { Группа ПРР РМЖ } \\
\text { Group of IRD of BC }\end{array}$ & $\begin{array}{l}\text { Общая группа } \\
\text { General group }\end{array}$ & $\begin{array}{l}\text { Групाа ПРР РМЖ } \\
\text { Group of IRD of BC }\end{array}$ \\
\hline $\begin{array}{l}\text { РМЖ } \\
\text { BC }\end{array}$ & 106 & 55 & $3,8 \pm 0,4$ & $5,1 \pm 0,7$ \\
\hline $\begin{array}{l}\text { ДЗМЖ } \\
\text { BBD }\end{array}$ & 47 & 24 & $4,7 \pm 0,7$ & $4,9 \pm 1,0$ \\
\hline
\end{tabular}

Примечание. ПРР - повышенный риск развития.

Note. IRD - increased risk of developing.

с использованием критерия $\chi^{2}$ с поправкой Йейтса. Различие частот считалось статистически значимым при $p<0,05$.

Стандартная ошибка частоты мутации $s p$ вычислялась по формуле

$$
s p=\operatorname{sqrt}(p(1-p) / n)
$$

где $p$ - частота мутации, $n-$ число пациентов в группе.

\section{РЕЗУЛЬТАТЫ И ОБСУЖДЕНИЕ}

В результате проведенного молекулярно-генетического обследования у 106 больных РМЖ (3,8 \%) и 47 пациенток с ДЗМЖ (4,7 \%) выявлена мутация c.470T $>$ С в гене $C H E K 2$ (табл. 2).

У больных РМЖ с данным генетическим вариантом средний возраст манифестации заболевания составил 52 года (табл. 3). Более половины из них (53 \%) к моменту обнаружения заболевания были старше 50 лет. Только 9 \% пациенток имели онкологически отягощенный семейный анамнез, 7 \% имели первичномножественные опухоли (РМЖ/РМЖ или РМЖ/РЯ). Средний возраст манифестации заболевания у носителей мутации с ДЗМЖ был ниже и составил 41 год. Только у половины из них (51 \%) наблюдался онкологически отягощенный семейный анамнез. В группах пациенток с мутацией признаки ПРР РМЖ отмечены примерно у половины обследованных (у 52 \% больных РМЖ и 51 \% пациенток с ДЗМЖ), что говорит об отсутствии связи между наличием данного генетического варианта у пациенток и присутствием характерных признаков ПРР заболевания.

Частота данного генетического варианта в группах повышенного риска составила 5,1 \% и 4,9 \% соответственно (см. табл. 2). Различий, имеющих статистическую значимость, между частотой мутации с.470Т >C в общих группах больных РМЖ и пациенток с ДЗМЖ и соответствующей частотой в группах повышенного риска, а также в группах больных РМЖ и пациенток с ДЗМЖ не установлено $(p>0,05)$. Данный факт можно объяснить как низкой вероятностью развития заболевания при наличии данного генотипа (низкой
Таблица 3. Клиническая характеристика пациентов с мутацией c. $470 T>$ С в гене CHEK2

Table 3. Clinical characteristics of patients with the c.470T $>$ C mutation in the CHEK2 gene

\begin{tabular}{|c|c|c|}
\hline \multirow{2}{*}{$\begin{array}{l}\text { Характеристика } \\
\text { Characteristic }\end{array}$} & \multicolumn{2}{|c|}{$\begin{array}{l}\text { Число пациентов, } \boldsymbol{n}(\%) \\
\text { Number of patients, } n \text { (\%) }\end{array}$} \\
\hline & $\begin{array}{c}\text { РМЖ, } \\
\boldsymbol{n}=\mathbf{1 0 6} \\
\mathrm{BC}, n=106\end{array}$ & $\begin{array}{c}\text { ДЗМЖ, } \\
n=47 \\
\mathrm{BBD}, n=47\end{array}$ \\
\hline \multicolumn{3}{|c|}{$\begin{array}{l}\text { 1. Boзраст } \\
1 . \text { Age }\end{array}$} \\
\hline $\begin{array}{l}\text { Средний возраст манифе- } \\
\text { стации заболевания } \\
\text { Average age of disease } \\
\text { manifestation }\end{array}$ & $\begin{array}{l}52(24-85) \text { года } \\
52(24-85) \text { years }\end{array}$ & $\begin{array}{l}41(26-61) \text { год } \\
41(26-61) \text { years }\end{array}$ \\
\hline $\begin{array}{l}\text { До } 50 \text { лет } \\
\text { Under } 50 \text { у.о. }\end{array}$ & $50(47)$ & 42 (89) \\
\hline $\begin{array}{l}51 \text { год и старше } \\
51 \text { у.o. and older }\end{array}$ & $56(53)$ & $5(11)$ \\
\hline \multicolumn{3}{|c|}{$\begin{array}{l}\text { 2. Семейный онкологический анамнез } \\
\text { 2. Pamily cancer history }\end{array}$} \\
\hline $\begin{array}{l}\text { Отягощен } \\
\text { Burden }\end{array}$ & $10(9)$ & $24(51)$ \\
\hline $\begin{array}{l}\text { Не отягощен } \\
\text { Not burden }\end{array}$ & $96(91)$ & 23 (49) \\
\hline
\end{tabular}

Not burden

\begin{tabular}{|c|c|c|}
\hline \multicolumn{3}{|c|}{$\begin{array}{l}\text { 3. Диагноз } \\
\text { 3. Diagnosis }\end{array}$} \\
\hline $\begin{array}{l}\text { ПМЗН (РМЖ/РМЖ } \\
\text { или РМЖ/РЯ) } \\
\text { PMМN (ВС/BC or BC/OC) }\end{array}$ & $7(7)$ & - \\
\hline $\begin{array}{l}\text { РMЖ } \\
\text { BC }\end{array}$ & 99 (93) & - \\
\hline
\end{tabular}

$\mathrm{BC}$

4. Признак повышенного риска развития РМЖ

Наличие

Presence

$55(52)$

Отсутствие

Absence

$51(48)$

Примечание. ДЗМЖ - доброкачественные заболевания молочной железы; ПМЗН - первично-множественные злокачественные новообразования; РМЖ - рак молочной железы; РЯ - рак яичников.

Note. $B B D$ - benign breast diseases; PMMN - multiple primary malignant neoplasms; $B C$ - breast cancer; $O C$ - ovarian cancer. 
пенетрантностью мутации), так и отсутствием связи между наличием данного генетического варианта и повышением риска развития онкологического заболевания.

В исследовании, проведенном в НМИЦ онкологии им. Н.Н. Блохина, частота мутации с.470Т $>$ С в гене $C H E K 2$ в группе больных РМЖ $(n=963)$ составила $5,7 \%$ [2], что в 1,5 раза превышает частоту генетического варианта, полученную в нашем исследовании $(95 \%$ доверительный интервал (ДИ): $1,08-2,16 ; p=0,01)$. Этот факт может объясняться гетерогенностью выборки по этнической принадлежности. Частота данной мутации, которая была установлена в российском исследовании у больных РМЖ из Республики Башкортостан $(n=977)$ [9], составила 5,12 \% и не имеет статистически значимых различий с соответствующей частотой, полученной в нашем исследовании $(p>0,05)$. Оба исследования, ранее проведенные в российской популяции [2, 9], показали отсутствие статистически значимых различий между частотой мутации с.470Т $>$ C в гене $C H E K 2$ в группе больных РМЖ и контрольной группе, что подтверждает предположение о вероятном отсутствии связи между наличием этого генетического варианта и повышением риска развития РМЖ.

Данные исследований зарубежных ученых носят противоречивый характер. С. Cybulski и O. Kilpivaara и соавт. показали, что в польской и финской популяциях наблюдается относительно небольшое повышение риска развития РМЖ у носителей мутации c. $470 \mathrm{~T}>\mathrm{C}$ в гене $C H E K 2$ (ОШ равно 1,4 и 1,43 соответственно) $[5,6]$.

Риск развития РМЖ оказался значительно повышен у носителей данного генетического варианта при обследовании немецкой и белорусской популяций (ОШ равно 3,6 и 4,5 соответственно) [4]. Метаанализ результатов обследований 19621 больного РМЖ и 27001 здорового донора, проведенных в ряде стран Европы и Америки (Польша, Финляндия, Чехия, Белоруссия, Нидерланды, Великобритания, США, Канада и Германия), показал, что наличие мутации с.470Т $>$ С в гене CHЕК2 сопряжено с ПРР РМЖ (ОШ = 1,48; 95 \% ДИ: $1,31-1,66 ; p<0,0001)$ [7]. Другой метаанализ результатов обследований 15985 больных РМЖ и 18609 здоровых доноров из Германии, Польши, Латвии, Чехии и Белоруссии выявил более высокий риск развития заболевания, ассоциированный с данным генетическим вариантом (ОШ = 1,58; 95 \% ДИ: 1,42-1,75, $p<0,00001)$ [8]. Зарубежные исследования опровергают связь между наличием мутации с.470T >C в гене CHEK2 и повышением риска развития РМЖ у жителей Северной Америки и Чехии [10-12]. В некоторых популяциях данный генетический вариант вовсе не был обнаружен. Мутация с.470T>C в гене CHEK2 отсутствовала у жителей Великобритании, Якутии (Россия), Китая и Ирана [10, 16-18].

\section{ЗАКЛЮЧЕНИЕ}

Таким образом, результаты проведенного исследования, а также других российских исследований свидетельствуют о вероятном отсутствии связи между наличием мутации с.470T $>$ С в гене $C H E K 2$ и повышением риска развития РМЖ. Однако в связи с наличием зарубежных исследований, подтверждающих связь данного генетического варианта с развитием РМЖ, проведение масштабных исследований для изучения вклада мутации с.470Т >C в риск развития онкологического заболевания является клинически важной и актуальной задачей.

\section{$\begin{array}{lllllllllllllllllllll}\text { Л И } & \text { T } & \text { E } & \text { P } & \text { A } & \text { I } & \text { У } & \text { P } & \text { A } & \text { I } & \text { R } & \text { E } & \text { F } & \text { E } & \text { R } & \text { E } & \text { N } & \text { C } & \text { E } & S\end{array}$}

1. Stracker T.H., Usui T., Petrini J.H. Taking the time to make important decisions: the checkpoint effector kinases Chk1 and Chk2 and the DNA damage response. DNA Repair 2009;8(9):1047-54 DOI: 10.1016/j.dnarep.2009.04.012.

2. Батенева Е.И. Новая диагностическая панель для выявления наследственной предрасположенности к развитию рака молочной железы и рака яичников. Дис. ... канд. мед. наук. ФГБНУ «Российский онкологический научный центр имени Н.Н.Блохина» Минздрава России. М., 2015. 125 c. [Bateneva E.I. A new diagnostic panel for detecting hereditary predisposition to breast cancer and ovarian cancer. Diss. ... candidate of medical sciences. N.N. Blokhin Russian Cancer Research Center of the Ministry of Health of the Russian. Moscow, 2015. 125 p. (In Russ.)].
3. Weischer M., Bojesen S.E., Ellervik C. et al. $C H E K 2^{*} 1100 \mathrm{delC}$ genotyping for clinical assessment of breast cancer risk: meta-analyses of 26,000 patient cases and 27,000 controls. J Clin Oncol 2008;26(4): 542-8. DOI: 10.1200/JCO.2007.12.5922.

4. Bogdanova N., Enssen-Dubrowinskaja N., Feshchenko S. et al. Association of two mutations in the CHEK2 gene with breast cancer. Int J Cancer 2005; 116:263-6. DOI: $10.1002 /$ ijc. 21022.

5. Cybulski C. Selected aspects of inherited susceptibility to prostate cancer and tumours of different site of origin. Hered Cancer Clin Pract 2007;5(3):164-79. DOI: 10.1186/1897-4287-5-3-164.

6. Kilpivaara O., Vahteristo P., Falck J. et al. CHEK2 variant I157T may be associated with increased breast cancer risk. Int J Cancer 2004;111:543-7. DOI: 10.1002/ijc.20299.
7. Liu C., Wang Y., Wang Q.S. et al. The CHEK2 I157T variant and breast cancer susceptibility: a systematic review and meta-analysis. Asian Pac J Cancer Prev 2012;13(4):1355-60. DOI: 10.7314/apjcp.2012.13.4.1355.

8. Han F.F., Guo C.L., Liu L.H. The effect of CHEK2 variant I157T on cancer susceptibility: evidence from a metaanalysis. DNA Cell Biol 2013;32(6):329-35. DOI: 10.1089/dna.2013.1970.

9. Бермишева М.А., Тахирова 3.Р., Богданова Н. и др. Частота мутаций в гене CHEK2 у больных раком молочной железы из Республики Башкортостан. Молекулярная биология. 2014;48(1):55-61. [Bermisheva M.A., Tahirova Z.R., Bogdanova N. et al. Frequency of $C H E K 2$ gene mutations in patients with breast cancer from the republic of bashkortostan. 
Molekuljarnaja biologija $=$ Molecular biology 2014;48(1):55-61. (In Russ.)] DOI: $10.7868 / \mathrm{S} 0026898414010029$.

10. Schutte M., Seal S., Barfoot R. et al. Variants in CHEK2 other than 1100delC do not make a major contribution to breast cancer susceptibility. Am J Hum Genet 2003;72(4):1023-8. DOI: 10.1086/373965.

11. Kleibl Z., Havranek O., Novotny J. et al. Analysis of CHEK2 FHA domain in Czech patients with sporadic breast cancer revealed distinct rare genetic alterations. Breast Cancer Res Treat 2008;112(1):159-64. DOI: 10.1007/s10549-007-9838-7.

12. Friedrichsen D.M., Malone K.E., Doody D.R. et al. Frequency of CHEK2 mutations in a population based, casecontrol study of breast cancer in young women. Breast Cancer Res 2004;6(6):629-35. DOI: $10.1186 /$ bcr933.

13. Daly M.B., Pilarski R., Berry M. et al. NCCN Guidelines Insights: Genetic/ Familial High-Risk Assessment: Breast and Ovarian, Version 2.2017. J Natl Compr

Canc Netw 2017;15(1):9-20.

DOI: 10.6004/jnccn.2017.0003.

14. Каприн А.Д., Костин А.А., Зикиряходжаев А.Д. и др. Рак молочной железы, ассоциированный с носительством мутации CHEK2. Акушерство и гинекология 2018;(5):102-7.

[Kaprin A.D., Kostin A.A.,

Zikyryahodzhayev A.D. et al. Breast cancer associated with carriage of a mutation CHEK2. Akusherstvo i ginekologija $=$ Obstetrics and gynecology 2018;(5):102-7. (In Russ.)]. DOI: 10.18565/aig.2018.5.102-7.

15. Paluch-Shimon S., Cardoso F., Sessa C. et al. Prevention and screening in BRCA mutation carriers and other breast/ovarian hereditary cancer syndromes: ESMO Clinical Practice Guidelines for cancer prevention and screening. Ann Oncol 2016;27(5):103-10. DOI: 10.1093/annonc/mdw327.
16. Фарахтдинова А., Федорова С., Николаева Т. и соавт. Анализ мутаций в генах BRCA1, CHEK2, NBS1 у больных раком молочной железы из Республики Саха (Якутия). Якутский медицинский журнал 2009;2(26):91-3. [Farahtdinova A., Fedorova S., Nikolaeva T. et al. Analiz mutacij v genah BRCA1, CHEK2, NBS1 u bol'nyh rakom molochnoj zhelezy iz Respubliki Saha(Yakutia). Jakutskij medicinskij zhurnal $=$ Yakut Medical Journal 2009;2(26):91-3. (In Russ.)].

17. Liu Y., Liao J., Xu Y. et al. A recurrent CHEK2 p.H371Y mutation is associated with breast cancer risk in chinese women. Hum Mutation 2011;32(9):1000-3. DOI: $10.1002 /$ humu. 21538 .

18. Jalilvand M., Oloomi M., Najafipour R. et al. An association study between CHEK2 gene mutations and susceptibility to breast cancer. Comp Clin Pathol 2017;26(4): 837-45.

DOI: $10.1007 / \mathrm{s} 00580-017-2455-\mathrm{x}$.

Вклад авторов:

Е.И. Новикова: сбор клинического материала, проведение молекулярно-генетических исследований, анализ и статистическая обработка результатов, подготовка и написание текста статьи;

В.К. Боженко: анализ результатов, редактирование текста статьи;

Е.А. Кудинова: анализ литературы, анализ результатов исследования;

В.А. Солодкий: планирование и анализ результатов исследования, редактирование текста статьи. Authors' contributions

E.I. Novikova: the collection of clinical material, carrying out molecular genetic studies, analysis and statistical processing of the results, preparation and writing of article;

V.K. Bozhenko: analysis of the results, edit the text of the article;

E.A. Kudinova: analysis of the literature, analysis of the research results;

V.A. Solodkiy: planning and analysis of the research results, edit the text of the article.

ORCID aвторов / ORCID of authors

Е.И. Новикова / Е.I. Novikova: https://orcid.org/0000-0003-4437-3661

B.К. Боженко / V.K. Bozhenko: https://orcid.org/0000-0001-8351-8152

Е.А. Кудинова / E.A. Kudinova: https://orcid.org/0000-0002-5530-0591

В.A. Солодкий / V.A. Solodkiy: https://orcid.org/ 0000-0002-1641-6452

Конфликт интересов. Авторы заявляют об отсутствии конфликта интересов.

Conflict of interest. The authors declare no conflict of interest.

Финансирование. Исследование проведено без спонсорской поддержки.

Financing. The study was performed without external funding.

Соблюдение прав пациентов и правил биоэтики:

Протокол исследования одобрен комитетом по биомедицинской этике ФГБУ «Российский научный центр рентгенорадиологии» Минздрава России (протокол № 3 от 27.03.2020 г.).

Все пациенты подписали информированное согласие на участие в исследовании.

Compliance with patient rights and principles of bioethics

The study protocol was approved by the biomedical ethics committee of Russian Scientific Center of Roentgenoradiology, Ministry of Health of Russia. All patients gave written informed consent to participate in the study.

Статья поступила: 11.12.2020. Принята к публикации: 09.03.2021.

Article submitted: 11.12.2020. Accepted for publication: 09.03.2021. 